\title{
Only a small population of adult Sertoli cells actively proliferates in culture
}

\author{
Andrey Yu Kulibin ${ }^{1, *}$ and Ekaterina A Malolina ${ }^{1,2, *}$ \\ ${ }^{1}$ Koltzov Institute of Developmental Biology of Russian Academy of Sciences, Moscow, The Russian Federation and \\ 2Ivanovsky Institute of Virology, N.F. Gamaleya Research Institute of Epidemiology and Microbiology, Ministry of \\ Health of the Russian Federation, Moscow, The Russian Federation
}

Correspondence should be addressed to A Yu Kulibin; Email: kulibin.a.bkrj@gmail.com

*(A Yu Kulibin and E A Malolina contributed equally to this work)

\begin{abstract}
Adult mammalian Sertoli cells (SCs) have been considered to be quiescent terminal differentiated cells for many years, but recently, proliferation of adult SCs was demonstrated in vitro and in vivo. We further examined mouse SC behavior in culture and found that there are two populations of adult SCs. The first population is SCs from seminiferous tubules that hardly proliferate in vitro. The second population is small and consists of SCs with atypical nuclear morphology from the terminal segments of seminiferous tubules, a transitional zone (TZ). TZ SCs multiply in culture and form colonies, display mixture of mature and immature SC characteristics, and generate cord-like structures in a collagen matrix. The specific features of TZ SCs are ACTA2 expression in vitro and DMRT1 low levels in vivo and in vitro. Although the in vivo function of TZ SCs still remains unclear, this finding has significant implications for our understanding of SC differentiation and functioning in adult mammals.
\end{abstract}

Reproduction (2016) 152 271-281

\section{Introduction}

It has been suggested for many years that Sertoli cells (SCs) in mammals do not proliferate after puberty (Cupp \& Skinner 2005). However, during the last decade, some in vivo and in vitro studies have shown that adult SCs can resume proliferation (Tarulli et al. 2012). Expression of proliferating cell nuclear antigen (PCNA) was demonstrated in SCs of seasonal breeding Djungarian hamsters after gonadotropin suppression and subsequent FSH replacement (Tarulli et al. 2006) and in SCs from healthy adult men after prolonged gonadotropin suppression (Tarulli et al. 2013). Proliferation of cultured adult mouse and human SCs was reported by Ahmed and colleges (2009). The authors showed that, as early as 3 days in culture, $10 \%$ of murine SCs incorporated $\mathrm{BrdU}$, and the number of BrdU-positive SCs reached 36\% after 20 days. SC proliferative activity was associated with a decrease in the cell cycle inhibitor p2 $7^{\text {kip } 1}$ expression and an increase in the proliferation inducer ID2 levels. Reinitiation of proliferation was also demonstrated in a primary SC culture of 20-day postpartum rats treated with activin (Nicholls et al. 2012). Therefore, it was concluded that adult SCs were not terminally differentiated cells, but a fine balance between cell cycle inducers and inhibitors exists in a postpubertal testis and prevents SCs from resuming proliferation.
Early light and electron microscopic studies on testes of different species (Dym 1974, Osman 1978, Nykänen 1979, Wrobel et al. 1986) demonstrated that the population of adult SCs is not homogeneous. So-called modified SCs were found in terminal segments of seminiferous tubules, connecting them with rete testis. According to terminology proposed by Dym (1974), this testis region was called the transitional zone (TZ), and the extension of rete testis connecting with it was called the tubulus rectus. Later, many authors (Naito et al. 2009, Aiyama et al. 2015) began to call TZ tubulus rectus, and the former tubulus rectus was classified as the rete testis tubule. In this study, we follow the earlier Dym's nomenclature.

In the TZ of seminiferous tubules, a gradual depletion of germ cells occurs, until finally tubules are lined only by SCs and a few spermatogonia (Dym 1974). Then, SCs form the terminal valve protruding into the tubulus rectus lined by a simple cuboidal or columnar epithelium. According to the electron microscopic study (Nykänen 1979), modified SCs of TZ have a multilobed nucleus with the typical nucleolus, but there is more peripheral heterochromatin than in SCs from seminiferous tubules, and nuclear lobulation seems to be more pronounced.

Early studies showed no evidence for proliferation of modified SCs in the adult testis; however, later it was observed that some unidentified cells of murine 
TZ incorporated BrdU after experimental autoimmune orchitis induction (Naito et al. 2009). Proliferation of some TZ SCs was clearly demonstrated in testes of adult Syrian hamsters under normal conditions (Aiyama et al. 2015). Moreover, some BrdU-positive SCs at 3 months after BrdU injection moved into the regions of seminiferous tubules with active spermatogenesis.

Summarizing the above findings, we hypothesized that adult SCs from seminiferous tubules (ST SCs) and modified TZ SCs should behave differently in culture. To test our hypothesis, we cultivated cells from TZ and ST separately and demonstrated that ST SCs hardly proliferate in vitro, whereas TZ SCs actively multiply, form colonies, display a mixture of mature and immature SC features, and generate seminiferous cordlike structures after embedding into a collagen matrix.

\section{Materials and methods}

\section{Animals}

Adult male $\mathrm{C} 57 \mathrm{BI} / 6 \mathrm{~J}$ mice that were $8-12$ weeks of age and mouse pups that were 4-6 days of age were used in the study. Animals were housed under standard conditions of care. All manipulations with animals were carried out in accordance
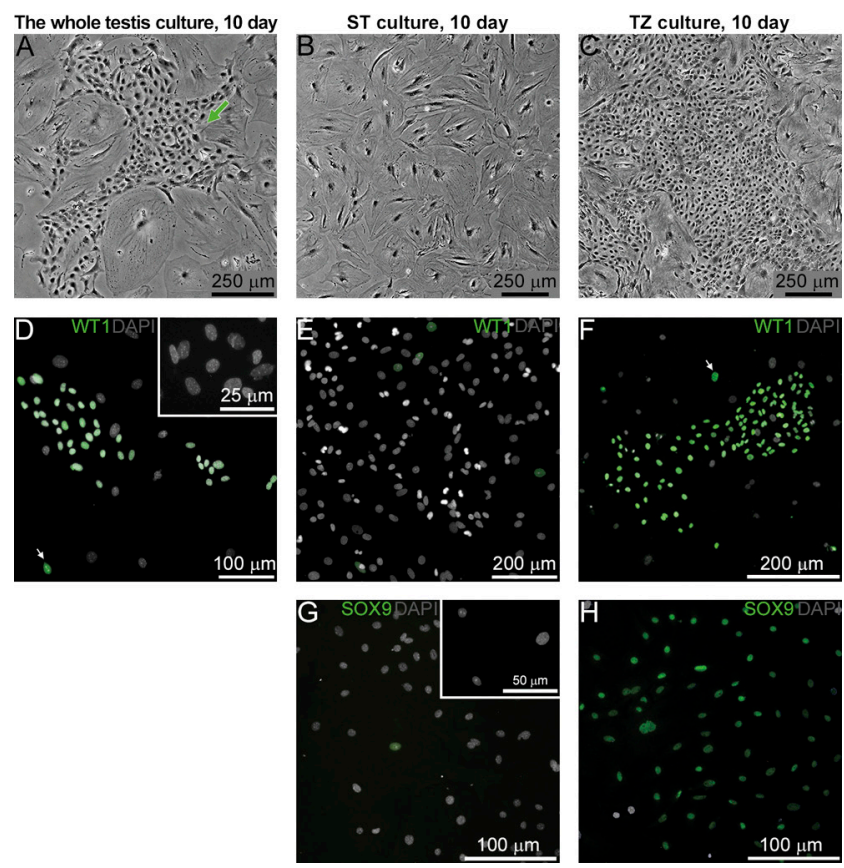

Figure 1 Adult mouse SCs isolated from the whole testis, ST, and TZ on day 10 of culture. (A-C) Phase contrast views of SC cultures. Colonies are present in the whole testis (A, arrow) and the TZ (C) cultures but not in the ST culture (B). Hereinafter, unless otherwise specified, images represent the $37 \_21$ culture.

(D-F) Anti-WT1 immunostaining of the whole testis (D), ST (E), and TZ (F) cultures; arrows indicate single $\mathrm{WT} 1^{+} \mathrm{SC}$ s outside the colonies in (D and F). ( $G$ and $H$ ) Anti-SOX9 immunostaining of the ST (G) and TZ $(H)$ cultures. Insets in (D and G) represent negative controls without first antibodies. with the National Institute of Health Guide for the Care and Use of Laboratory Animals (NIH Publications No. 80-23) revised 1996 and the UK Animals (Scientific Procedures) Act 1986 and were approved by the Animal Care and Use Committee of Koltzov Institute of Developmental Biology RAS.

\section{SC isolation and culture}

SCs were isolated either from the whole adult mouse testis or separately from ST and from TZ. In the last case, a testis was cut into two parts. One part was approximately a quarter of the testis around the vascular pedicle and contained rete testis, tubuli recti, TZ, and fragments of seminiferous tubules; another part contained only seminiferous tubules. Enzymatic digestion was performed according to Ahmed et al. (2009) with modifications described in the Supplementary Materials and methods, see section on supplementary data given at the end of this article. Cells were plated on culture dishes in alphaMEM/F12 medium (PanEco, Moscow, Russia) with 5\% (v/v) FBS (HyClone; Logan, UT, USA) at a concentration of $2.0 \times 10^{5} \mathrm{cells} / \mathrm{cm}^{2}$ (for the whole testis and ST isolation) and $1.0 \times 10^{5} \mathrm{cells} / \mathrm{cm}^{2}$ (for TZ isolation). The dishes were maintained either at $37^{\circ} \mathrm{C}$ under standard $21 \% \mathrm{O}_{2}$ atmosphere $\left(37 \_21\right)$ or at $34^{\circ} \mathrm{C}$ under lower $5 \% \mathrm{O}_{2}$ culture atmosphere (34_5). After $24 \mathrm{~h}$, the culture was washed from unattached germ cells and fresh medium containing $1 \%$ FBS was added; the medium was then changed every 3 days.

\section{$3 D$ cell culture in a collagen matrix}

SCs isolated from TZ were harvested using a trypsin solution after 10 days in culture and mixed at a ratio of 3:7 (Sang et al. 1990) with peritubular myoid cells (PMCs) obtained from the supernatant fraction after trypsin digestion of adult ST and cultivated with $10 \%$ FBS at $37^{\circ} \mathrm{C}$ under $21 \% \mathrm{O}_{2}$ for 3 days. Cells were embedded into a collagen matrix according to Zhang et al. (2014) with some modifications described in the Supplementary Materials and methods and maintained in 3D culture at $34^{\circ} \mathrm{C}$ under $5 \% \mathrm{O}_{2}$ culture atmosphere for 7 days. As a positive control, freshly isolated pup testicular cells or pup SCs cultured for 10 days were used, as described in the Supplementary Materials and methods. As a negative control, ST SCs were embedded into a collagen.

\section{Immunofluorescence and histology}

Immunofluorescence staining of testis sections, cultured cells, and cell aggregates in a collagen matrix was performed according to protocols described in the Supplementary Materials and methods. Samples were photographed using a Keyence

Table 1 The percentage of $\mathrm{WT} 1^{+}$cells in the adult mouse SC cultures on day 10.

\begin{tabular}{lclc}
\hline Culture & The whole testis & ST & TZ \\
\hline $37 \_21(n=5)$ & $37.0 \pm 10.1$ & Sporadic cells & $80.8 \pm 2.8^{\mathrm{a}}$ \\
34_5 $(n=7)$ & $45.9 \pm 8.8$ & Sporadic cells & $50.1 \pm 5.8^{\mathrm{b}}$ \\
\hline
\end{tabular}

a $P<0.05$ compared with the 37_21 whole testis culture, ${ }^{b} P<0.05$ compared to the 37_21 TZ culture (Mann-Whitney $U$ test; data represent mean \pm S.E.M.). 
A

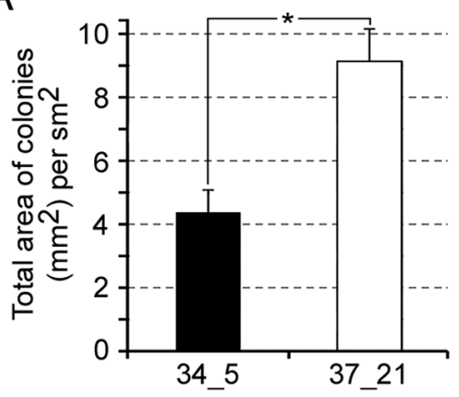

D

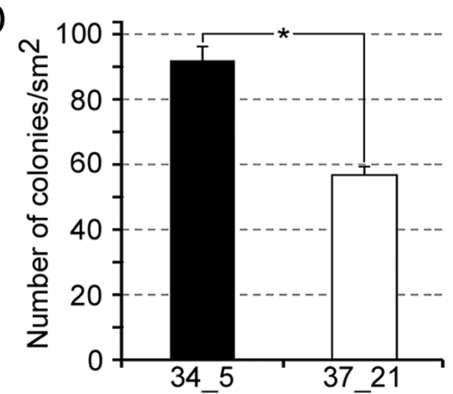

E

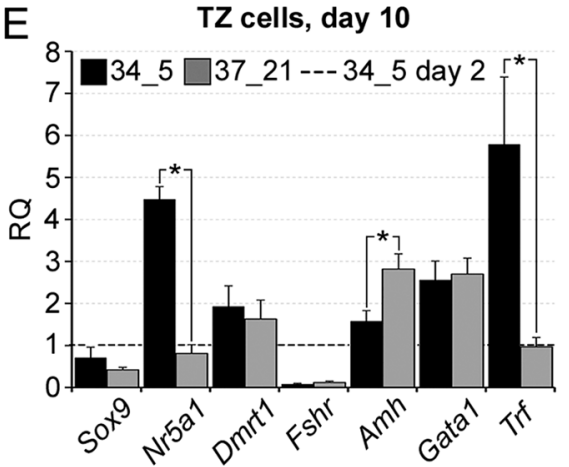

F TZ cells, passage 2 (37_21)

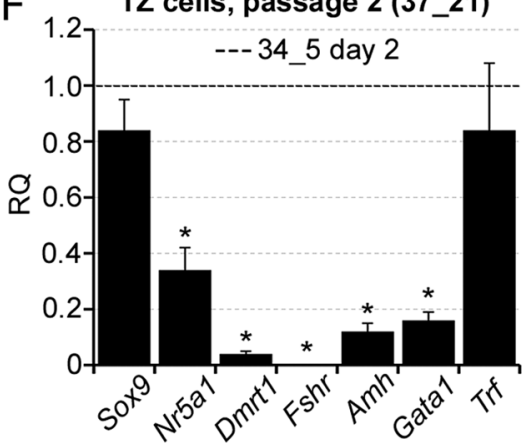

B

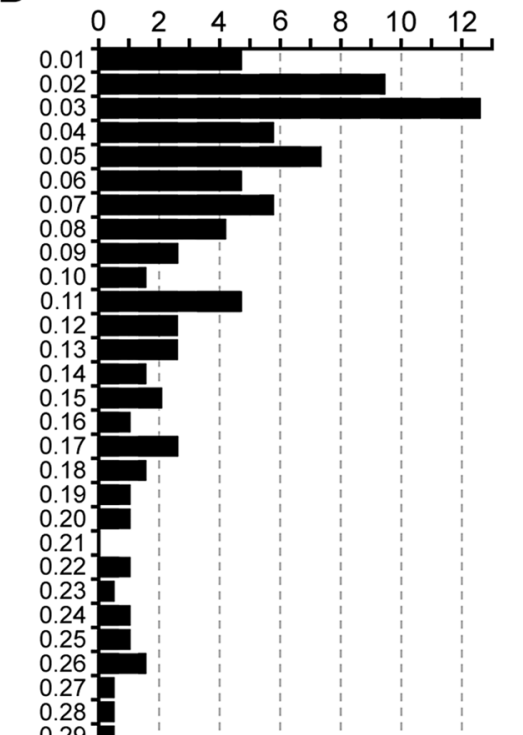

C

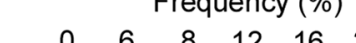

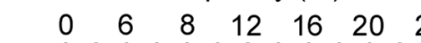

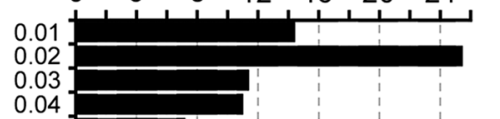

0.04

0.05

0.06
0.07
0.09

0.08

0.09

0.11

0.12

0.13

0.14

0.15

$0.16]$

0.17

$0.19\}$

0.20

0.21

0.22

0.24

0.25

0.26

0.28

तิ 0.30

ก 0.30

है 0.30

है 0.32

s 0.33

क 0.34

등 0.35

0.37
0

ㄴ 0.38 .

$\begin{array}{ll} & 0.39 \\ & 0.40\end{array}$

) 0.40

这 0.41

0.42
0.43

0.44

0.45

$0.46-$

0.46
0.47
0.48

0.48
0.49
0.50

0.49
0.50

0.50

0.51
0.52
0.53

0.53 .

0.54

0.807

0.81 -

0.82

0.83

0.84

0.85

0.86

0.87
0.88
0.89

\begin{tabular}{l}
0.88 \\
0.89 \\
\hline
\end{tabular}

0.89 -

$1.61 \mathrm{~J}$

37_21

$1.62-$

$2.01 \mathrm{~J}$

34_5

190 colonies
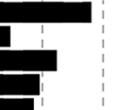

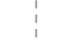

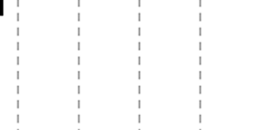

$$
2.02 \mathrm{~J}
$$

263 colonies

Figure 2 Quantitative analysis of colonies and qRT-PCR assay in the TZ culture. (A) A graph of the total colony area per sm² vs culture type. (B, C) Histograms of the individual colony area distributions in the 37_21 (B) and 34_5 (C) cultures summed from three independent experiments. (D) A graph of the colony number per $\mathrm{sm}^{2}$ vs culture type. Bars in (A) and (D) indicate mean \pm S.E.M; ${ }^{*} P<0.05$ (Mann-Whitney $U$ test, $n=3$ ). (E, F) qRT-PCR assay for the indicated genes in the 34_5 and 37_21 TZ cultures on day 10 (E), and at passage 2 of the $37 \_21$ TZ culture (F). Relative quantification of RNA (RQ) was normalized with Wt1. Bars indicate mean \pm s.E.M.; dotted lines represent levels of gene expression in the TZ culture on day 2 (34_5 culture). ${ }^{*} P<0.05$ (Mann-Whitney $U$ test, $n=3$ ).

(Model BZ-9000, Osaka, Japan) or an Olympus (IX51, Japan) microscopes and a confocal microscope (TCS SP5 STED; Leica Microsystems, Wetzlar, Germany). For histology, testes were fixed with modified Davidson's fluid for $24 \mathrm{~h}$ and embedded in paraffin; sections $(7 \mu \mathrm{m})$ were stained with Caracci's hematoxylin and eosin and photographed using a Keyence microscope. 


\section{BrdU labeling}

For in vivo BrdU labeling, a BrdU solution $(0.1 \mathrm{mg} / \mathrm{g}$ body weight; Sigma) was injected intraperitoneally into five adult male mice once per day for 5 days. Mice were killed and a testis from each mouse was isolated and snap-frozen on day 1 after the last BrdU injection. Three frozen sections were cut from a rete testis zone of each testis with an interval of $80 \mu \mathrm{m}$ between sections, double stained for GATA4 and BrdU, photographed using a Keyence microscope, and analyzed. For in vitro labeling, BrdU $(3 \mu \mathrm{g} / \mathrm{mL})$ was added to the cell culture.

\section{Analysis of colonies in the TZ cultures}

TZ cells were photographed under phase contrast using a Keyence microscope on day 10 of culture. Image processing was performed with Image (NIH) software. The number of colonies, their total area, and the areas of individual colonies were measured. Live imaging of colony formation for the 37_21 TZ cultures was also performed; the procedure is described in the Supplementary Materials and methods.

\section{DNA image cytometry}

Cultures of cells isolated from ST and TZ were immunostained for WT1 on day 10 of culture. Nuclear DNA was stained with propidium iodide (PI, Sigma). Cells were photographed using an Olympus microscope. Then, the integrated PI staining intensity was measured by automated image analysis (CellProfiler software), and DNA content histograms were built, as described (Carpenter et al. 2006) (Supplementary Fig. 1). The percentage of WT1-positive cells with $2 \mathrm{~N}, 2-4 \mathrm{~N}$ and $>4 \mathrm{~N}$ DNA content was calculated.

\section{RNA isolation and $q R T-P C R$}

Total RNA was extracted from samples using the RNeasy Mini Kit (Qiagen) and reverse-transcribed using the MMLV
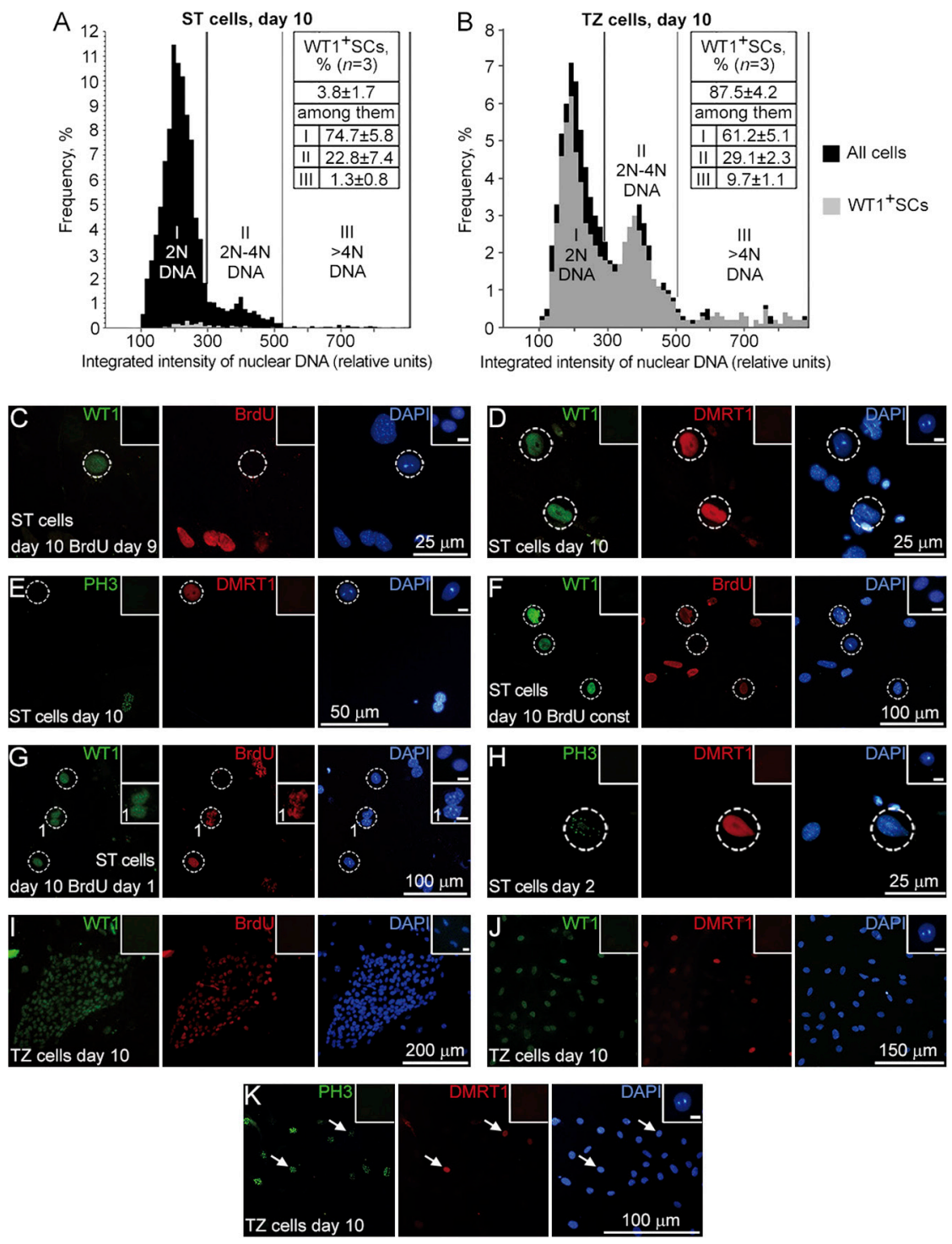

Figure 3 Proliferation of ST and TZ SCs in culture. (A and B) Representative histograms of DNA content in ST (A) and TZ (B) cultures built using CellProfiler. (C-K) Double immunostaining of the ST $(\mathrm{C}-\mathrm{H})$ and the TZ (I-K) cultures for WT1 and BrdU (C, F, G, and I), for WT1 and DMRT1 (D and J), and for WT1 and PH3 (E, H, and K). Stainings were performed on days 10 ( $\mathrm{C}-\mathrm{G}$ and $\mathrm{I}-\mathrm{K})$ and 2 $(\mathrm{H})$. BrdU was added to the cultures on day 9 (C and I), once per day from day 1 to day 9 $(\mathrm{F})$, or on day $1(\mathrm{G})$. Inset 1 represents an enlarged view of SCs with punctate BrdU staining (G). Dashed circles outline ST SCs. Arrows point to $\mathrm{Dmrt} 1^{+} / \mathrm{PH} 3^{+} \mathrm{TZ} \mathrm{SCs}$ in (K). Insets represent negative controls without first antibodies. Scale bars in all insets: $10 \mu \mathrm{m}$. 
Table 2 The percentage of $\mathrm{WT}^{+}$and BrdU ${ }^{+}$cells in the adult mouse TZ and ST cultures on day 6.

\begin{tabular}{lccccc}
\hline Culture type & & WT1 ${ }^{+}$cells & BrdU $^{+}$cells & WT1 $^{+}$BrdU $^{+}$cells & WT1 $^{+}$BrdU $^{+} / \mathbf{W T 1}^{+}$cells \\
TZ & $37 \_21(n=3)$ & $47.2 \pm 4.6$ & $47.1 \pm 7.3$ & $26.8 \pm 6.4$ & $56.0 \pm 11.0$ \\
& $34 \_5(n=3)$ & $12.0 \pm 1.8^{\mathrm{a}}$ & $41.8 \pm 1.0$ & $9.6 \pm 2.4^{\mathrm{a}}$ & $77.5 \pm 9.6$ \\
ST & $37 \_21(n=3)$ & $3.8 \pm 0.7^{\mathrm{a}}$ & $47.0 \pm 5.2$ & $0^{\mathrm{a}}$ & $0^{\mathrm{a}}$ \\
& $34 \_5(n=3)$ & $4.7 \pm 0.9^{\mathrm{b}}$ & $32.7 \pm 3.1^{\mathrm{b}}$ & $0^{\mathrm{b}}$ & $0^{\mathrm{b}}$ \\
\hline
\end{tabular}

${ }^{a} P<0.05$ compared with 37_21 TZ culture, ${ }^{b} P<0.05$ compared with 34_21 TZ culture (Mann-Whitney $U$ test; data represent mean \pm S.E.M.).

RT kit (Evrogen, Moscow, Russia). Oligonucleotide primers (Supplementary Table) were ordered from Evrogen. Real-time PCR analysis was performed in triplicates using SYBR green qPCRmix-HS with ROX (Evrogen) with a StepOnePlus RealTime PCR System (Applied Biosystems). Mean $C_{t}$ values for Wt1 (for SC-specific genes) and Hprt (for Acta2, Snai1 and Twist1) were used to calculate $\Delta C_{t}$ values for each sample. Wt1 is considered to be a stable SC marker and, according to our immunofluorescent data, it is continuously expressed in SCs throughout culture time. The melting curves for Wt1, Hprt. and genes analyzed gave only a single, unique peak for each primer set. $\Delta \Delta \mathrm{C}_{\mathrm{t}}$ values for each sample were calculated by subtracting the mean $\Delta \mathrm{C}_{\mathrm{t}}$ of the $34 \_5 \mathrm{TZ}$ culture on day 2 , except Snai1 and Twist 1 for which the mean $\Delta C_{t}$ of cultured mouse embryonic fibroblasts (MEF) was used. Relative quantification of RNA (RQ) was calculated using the $2^{-\Delta \Delta C t}$ method (Livak \& Schmittgen 2001).

\section{Statistical analysis}

All quantitative data were presented as mean \pm S.E.M. (n represents independent experiments) and analyzed using the non-parametric Mann-Whitney $U$ test.

\section{Results}

\section{There are two SC populations in an adult testis: $S T, T Z S C s$}

We cultured SCs isolated from the whole testis and SCs isolated separately from ST and TZ. After 10 days, cells from all cultures formed a subconfluent monolayer, which in the case of the whole testis isolation consisted of large irregular-shaped cells and colonies of smaller cells (Fig. 1A, arrow). Cells obtained from ST formed a monolayer consisting of large polygonal cells, and no colonies were present (Fig. 1B). By contrast, cells from TZ formed large colonies (Fig. 1C). All cells from all cultures were positive for vimentin and GATA4, markers of somatic testicular cells (data not shown). After staining for a SC marker WT1, it was demonstrated that cells in colonies from the whole testis culture (Fig. 1D) and from the TZ culture (Fig. 1F) were WT1 ${ }^{+S C s}$. A few WT1 ${ }^{+}$SCs were also detected outside the colonies (Fig. 1D and F, arrows). WT1 ${ }^{+}$SCs were rarely detected in the ST culture and always lied separately (Fig. 1E). The same results were obtained after immunostaining the ST and the TZ cultures for another SC marker SOX9 (Fig. 1G and H). The specificity of the WT1 and SOX9 antibodies was confirmed by using mouse testis sections as positive controls (Supplementary Fig. 2A and C).

We maintained SCs at two culture conditions: at $37^{\circ} \mathrm{C}$ in an atmosphere of $21 \% \mathrm{O}_{2}$ and $5 \% \mathrm{CO}_{2}$ (37_21, standard conditions) or under lowered temperature $\left(34^{\circ} \mathrm{C}\right.$ ) and oxygen (5\%). The last conditions (34_5) are considered to be more physiological for testes (Harrison \& Weiner 1949, Vaupel et al. 1989). No significant difference between the 37_21 and 34_5 whole testis cultures was observed in the number of $\mathrm{WT}^{+}{ }^{+}$cells on day 10 (Table 1 ). By contrast, the number of WT1 ${ }^{+}$cells in the 37_21 TZ culture was 1.6-fold higher than in the 34 5 TZ culture, and in some cases, it reached $85 \%$. The 37 21 TZ culture also demonstrated a >twofold increase in the total area of colonies compared with the 34_5
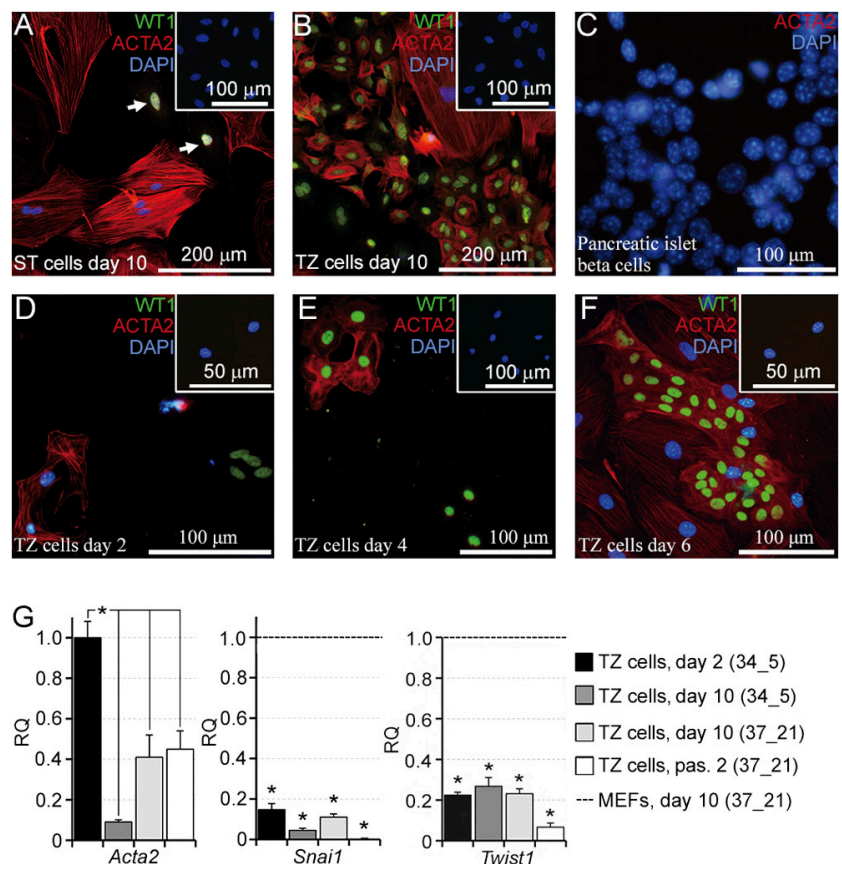

TZ cells, day $2(345)$ $\square T Z$ cells, day 10 (34_5) $\square$ TZ cells, day 10 (37_21) $\square$ TZ cells, pas. 2 (37_21)

Figure 4 ACTA2 expression in SC cultures. (A and B) Double immunostaining of the ST (A) and TZ (B) cultures for ACTA2 and WT1 on day 10; arrows point to WT1+/ACTA2-SCs in the ST culture. (C) ACTA2 staining of pancreatic $\beta$ cell line TC-6 (ATCC, CRL-11506, Manassas, VA, USA) for negative control. (D-F) Double immunostaining of the TZ culture for ACTA2 and WT1 on day 2 (D), day $4(\mathrm{E})$, and day $6(\mathrm{~F})$. Insets represent negative controls without first antibodies. (G) qRT-PCR analysis for Acta2, Snai1, and Twist1 in TZ cells. Relative quantification of RNA (RQ) was normalized with the reference gene encoding Hprt. Bars indicate mean \pm S.E.M. Dotted lines indicate levels of gene expression in MEFs. ${ }^{*} P<0.05$ (Mann-Whitney $U$ test, $n=3$ ). 
culture (Fig. 2A). Size distribution histograms of colonies (Fig. 2B and C) show that colonies in the 37_21 TZ culture were larger than in the 34_5 culture. However, the number of colonies per $\mathrm{sm}^{2}$ in the 34_5 TZ culture was 1.5-fold higher than in the 37_21 culture (Fig. 2D).

To further compare two types of culture conditions, the expression of some SC genes was measured in the TZ cultures on day 10 (Fig. 2E), the 34_5 TZ culture on day 2 was used as control. The TZ SCs were found to express all key SC transcriptional factors (Buganim et al. 2012): Wt1, Sox9, Nr5a1 and Dmrt1 as well as mature (Gata1 and Trf) and immature (Amh) SC markers. The FSH receptor (Fshr) expression was inhibited in both the cultures. The Nr5a1 and Trf expressions were
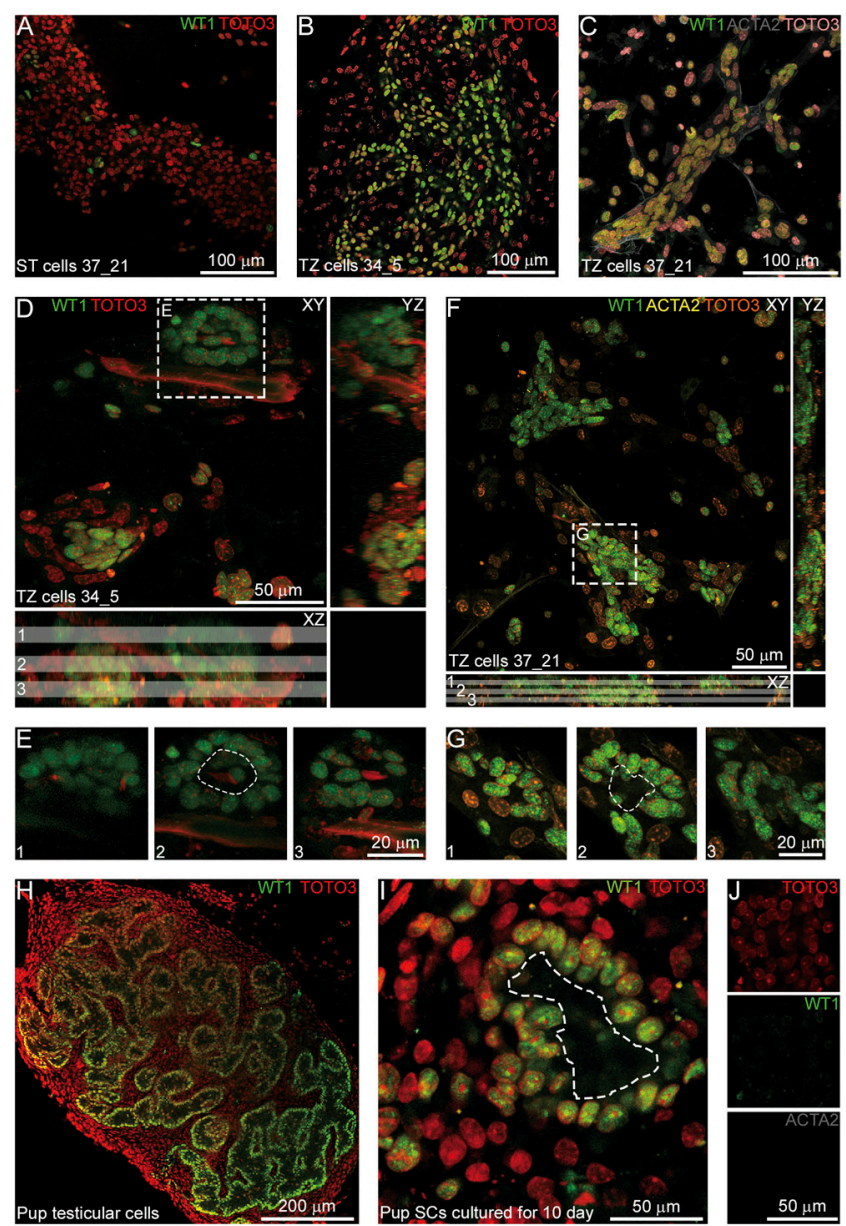

Figure 5 3D culture of SCs in a collagen matrix. (A) The 37_21 ST culture maintained in a collagen for 1 week, no cord-like structures were present. (B-I) Cord-like structures formed by TZ SCs from the 34_5 (B, D, and E) and 37_21 (C, F, and G) cultures, freshly isolated pup testicular cells $(\mathrm{H})$, and cultured pup SCs (I) that were maintained in a collagen for 1 week. Samples were immunostained for WT1 (A, B, D, E, H, and I) or for WT1 and ACTA2 (C, F, and G), nuclei were co-stained with TOTO3. Then, confocal microscopy was performed. In ( $\mathrm{D}$ and $\mathrm{F})$, the right and bottom panels represent orthogonal projections; optical sections (1-3) of areas denoted by dashed boxes are shown in ( $E$ and $G$ ). Dashed lines mark borders of cavities. (J) A negative control without first antibodies. upregulated, whereas Amh was slightly downregulated in the $34 \_5$ culture compared with the 37_21 culture. At passage 2 , on day 26 of culture, when $95.1 \pm 5.3 \%$ cells were WT1+SCs, Nr5a1, Dmrt1, Fshr, Amh and Gata1 expressions decreased dramatically (Fig. 2F), whereas Sox9 and Trf levels were only insignificantly changed.

\section{ST SCs hardly proliferate in vitro, whereas TZ SCS proliferate actively}

To estimate the proliferative capacity of ST and TZ SCs, DNA image cytometry was performed on day 10 of culture. Representative histograms of DNA content (Fig. $3 \mathrm{~A}$ and B) demonstrate that $22.8 \pm 7.4 \%$ of ST SCs and $29.1 \pm 2.3 \%$ of TZ SCs contained DNA in the range between $2 \mathrm{~N}$ and $4 \mathrm{~N}$. However, when BrdU was added to the ST culture on day 9, and cells were double stained for WT1 and BrdU after 18h, there were not BrdUpositive SCs (Fig. 3C). Moreover, no ST SCs, identified by
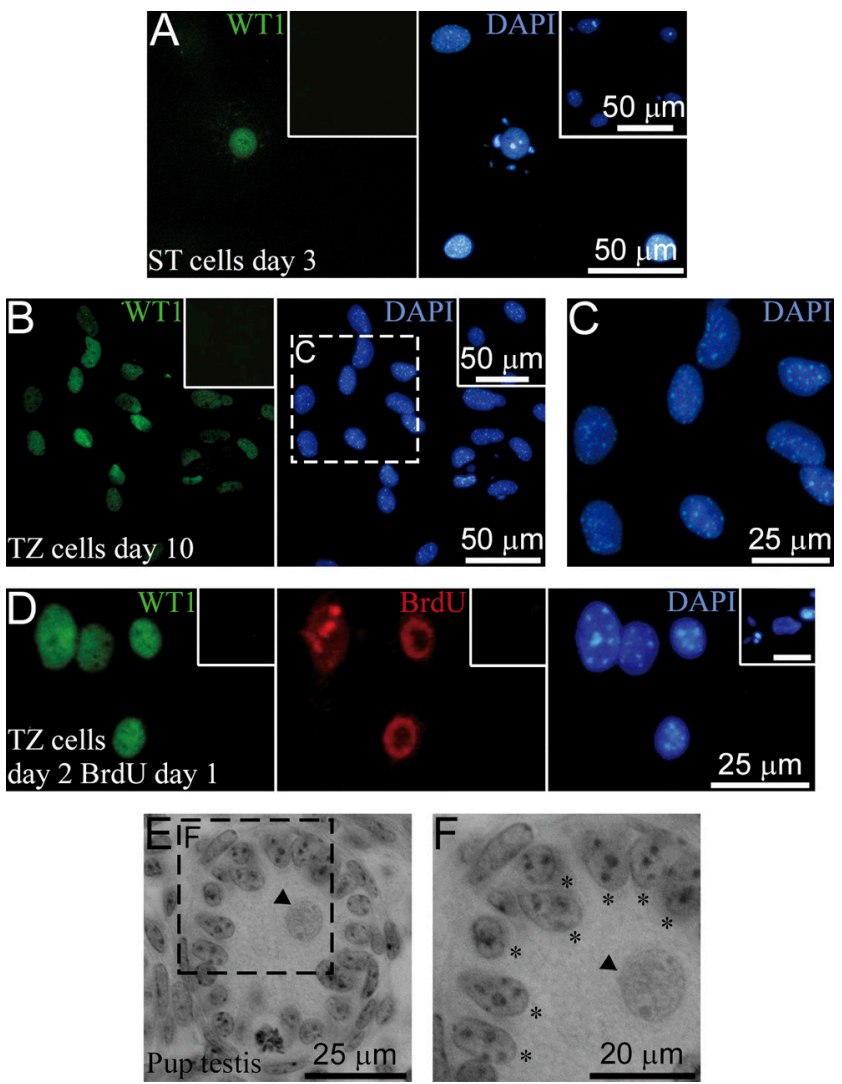

Figure 6 SC nuclear morphology in vitro. (A) A WT1+ ST SC with typical nuclear morphology on day 3 of culture. (B) A colony fragment of $\mathrm{WT} 1^{+} \mathrm{TZ}$ SCs with atypical nuclei on day 10 of culture. (C) An enlarged image of an area denoted by the dash box in (B). (D) $\mathrm{WT} 1^{+} / \mathrm{BrdU}^{+}$cells have atypical nuclei with several heterochromatin clusters on day 2 of culture. (E) A seminiferous tubule from a 4-day-old mouse pup testis, hematoxylin and eosin staining. (F) An enlarged image of an area denoted by the dash box in (E). Arrowheads point to a gonocyte, asterisks indicate SC nuclei. Insets in $(A, B$, and $D)$ represent negative controls without first antibodies, a scale bar in (D): $10 \mu \mathrm{m}$. 
immunostaining for another SC marker DMRT1 (Fig. 3D), were positive on the phosphohistone $\mathrm{H} 3-\mathrm{Ser} 10(\mathrm{PH} 3)$, mitosis marker (Fig. 3E). Hence, it is likely that 2-4N ST SCs represent cell cycle-arrested cells.

To further investigate the ST SC cell cycle, BrdU was added to the ST culture once per day from day 1 to day 9 , and immunostaining was performed on day 10 . Some SCs were found to be BrdU positive (Fig. 3F), but most SCs did not incorporate BrdU and remained quiescent throughout the culture period. When cells were treated with $\mathrm{BrdU}$ for $18 \mathrm{~h}$ from day 1 to day 2 of culture and immunostained on day 10 , most of the WT1- cells had punctate BrdU staining because of the label dilution during cell divisions after BrdU incorporation; however, SCs were stained solidly, and only one pair of SCs with punctate staining was observed (Fig. 3G, 1). No $\mathrm{WT}^{+}{ }^{+}$ $\mathrm{BrdU}^{+}$cells were detected in the 34_5 and 37_21 ST cultures when BrdU was added to the culture on days 3 and 5 and immunostainings were performed on days 4 (data not shown) and 6 (Table 2) correspondingly. $\mathrm{PH}^{+}{ }^{+} \mathrm{SCs}$ were present only on days 2 and 3 (Fig. $3 \mathrm{H}$ ). These data gave evidence that ST SCs did not proliferate more than once (or perhaps twice) and that DNA synthesis took place within the narrow time interval from day 1 to day 3 of culture. The same results were obtained when SCs were maintained in $10 \%$ FBS, so the serum did not affect their proliferation.

As opposed to ST SCs, TZ SCs actively incorporated BrdU on day 10 (Fig. 3I). Hence, 2-4N TZ SCs (Fig. 3B) represent cells in the $\mathrm{S}$-phase. To investigate the difference between the two types of culture conditions, a BrdU incorporation assay was performed on day 6 at about $50 \%$ confluence. The percentage of $\mathrm{WT}^{+} / \mathrm{BrdU}^{+}$ cells as well as the percentage of $\mathrm{WT}^{+}{ }^{+}$cells were significantly higher in the 37_21 culture compared with the 34_5 culture (Table 2). However, the percentage of $\mathrm{BrdU}^{+}$cells among $\mathrm{WT}^{+}$cells was higher in the $34 \_5$ culture, although not significantly (Table 2). It is interesting that some cells in colonies were DMRT1 negative, and other cells expressed DMRT1 in a low level, although all of them expressed WT1 (Fig. 3J). Nevertheless, the presence of Dmrt1 ${ }^{+} / \mathrm{PH}^{+}$cells in the TZ colonies (Fig. 3K) clearly demonstrates that TZ SCs are able to enter mitosis, and as opposed to ST SCs, not cell cycle arrested on day 10 .

\section{TZ SCs begin to express a-smooth muscle actin in culture}

Double immunostaining for WT1 and $\alpha$-smooth muscle actin (ACTA2), a marker of PMCs, was performed on day 10. Most cells in the ST culture were found to be WT1-/ACTA2+PMCs; WT1+ SCs did not express ACTA2 (Fig. 4A, arrows). In the TZ culture, most cells outside the colonies, except a few WT1+/ACTA2-SCs, were WT1-/ACTA2+PMCs (Fig. 4B). Strikingly, most $\mathrm{WT} 1{ }^{+} \mathrm{SCs}$ in colonies were also positive for ACTA2
(Fig. 4B). The specificity of ACTA2 antibodies used was verified by staining pancreatic islet $\beta$ cells that did not express ACTA2 as a negative control (Fig. 4C) and staining a mouse testis section as a positive control (Supplementary Fig. 2B).

We observed that all $\mathrm{WT}^{+}{ }^{+}$cells in the TZ culture were ACTA2 negative on day 2 (Fig. 4D), but on day 4, some WT1 ${ }^{+}$cells began to express ACTA2 (Fig. 4E), and on day 6 , most WT1 ${ }^{+}$colonies were stained for it (Fig. 4F). However, according to the PCR analysis, Acta2 mRNA in the TZ cultures on day 10 and after passaging was significantly downregulated compared with the TZ culture on day 2 (Fig. 4G). As Acta2 is a mesenchymal marker and could be evidence of
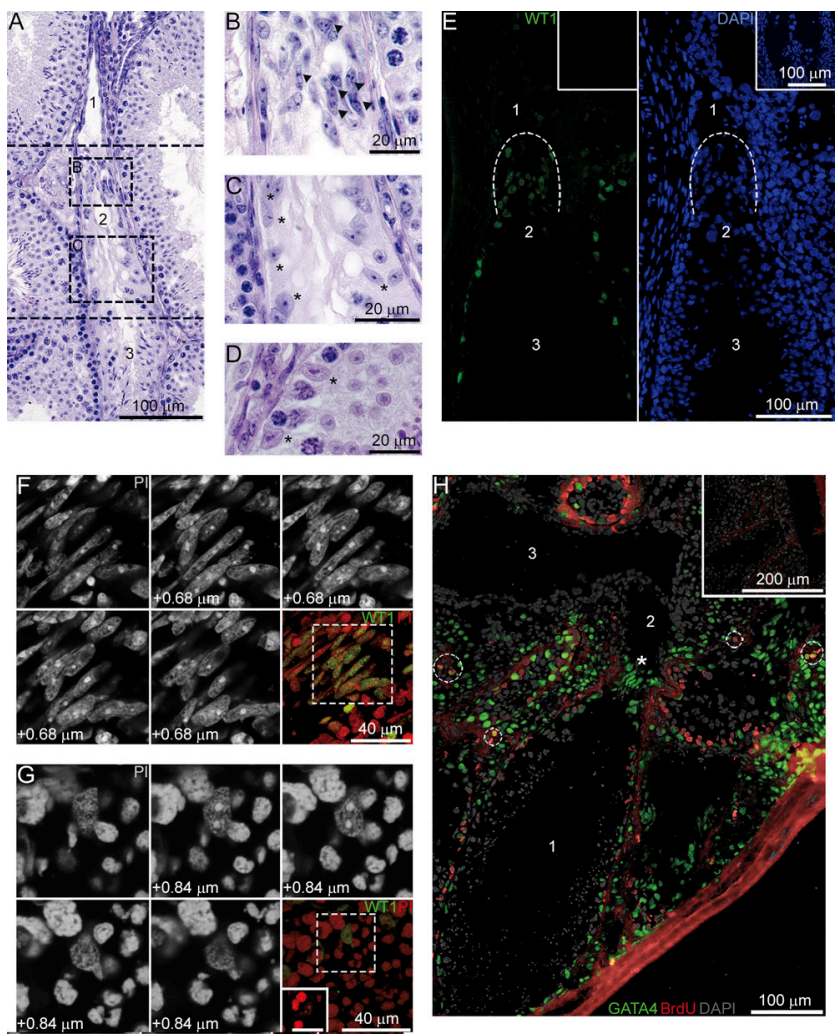

Figure 7 Morphological and immunofluorescence characterization of the TZ in the adult mouse testis. (A) TZ (2), a terminal segment of a seminiferous tubule (3), TZ is adjacent to a tubulus rectus (1), hematoxylin and eosin staining. (B and C) Enlarged images of areas in the TZ denoted by dash boxes in (A). (D) An enlarged image of the seminiferous tubule. Arrowheads point to modified SCs; asterisks mark typical SCs in (B-D). (E) Anti-WT1 immunostaining of the TZ, dashed curves indicate the proximal end of the TZ. WT1 expression is absent in the tubulus rectus (1). ( $F$ and G) Serial confocal images of modified SC nuclei (F) and a typical SC nucleus (G) stained with PI. The last images represent maximum projection views of these cells, positively stained for WT1 (surrounded by dash boxes). (H) Double immunostaining of the TZ for GATA4 and BrdU. 1 - seminiferous tubule, 2 - tubulus rectus, 3 - rete testis, an asterisk marks the TZ, dashed circles outline GATA4 ${ }^{+} / \mathrm{BrdU}^{+}$cells in the testis interstitium. Insets in $(\mathrm{E}, \mathrm{G}$, and $\mathrm{H})$ represent negative controls without first antibodies; a scale bar in (G): $15 \mu \mathrm{m}$. 
epithelial to mesenchymal transition (EMT), Snai1 and Twist1, two markers of EMT, were analyzed in cultures (Fig. 4G). However, the low level of their expression in the TZ cultures on days 2, 10, and after passaging was observed compared with the MEFs.

\section{TZ SCs organize into cord-like structures in the $3 D$ culture}

It is well known that dissociated immature testicular cells can reaggregate and form seminiferous tubule- or cordlike structures. To test whether SCs can form testicular cords, we mixed them with an additional number of PMCs, which contribute to cord formation as well and embedded them in a collagen matrix. The ST cultures did not form any structures after a week (Fig. 5A). By contrast, TZ SCs from the 34_5 (Fig. 5B) and the 37_21 (Fig. 5C) cultures were organized into $\mathrm{WT}^{+}{ }^{+}$cord-like structures. They often were surrounded by ACTA2+PMCs (Fig. 5C). In many cases, these structures resembled seminiferous tubules (Fig. 5D and F, dashed boxes). Serial optical sections (Fig. 5E and G) collected by using confocal microscopy showed the presence of a cavity inside.

All observed SC aggregates were small and did not form a network of elongated anastomotic seminiferous cords that the freshly isolated pup testicular cells generated (Fig. 5H). However, pup SCs cultured for 10 days behaved like TZ SCs and were able to form only small cord-like structures (Fig. 5I). Therefore, it is possible that other testicular cell populations (i.e., endothelial cells), which were present among pup cells, were needed for efficient cord formation as it occurs during embryonal testis development (Cool et al. 2012). It could also be presumed that SCs lose some essential characteristics during culturing, and more optimal culture conditions should be found.

\section{TZ SCs have atypical nuclear morphology in vitro and in vivo}

Most ST SCs in culture, except those entering the cell cycle, displayed typical adult SC nuclear morphology with two large DAPI bright perinucleolar chromocenters (Fig. 6A). However, TZ SCs in colonies had atypical nuclei with many smaller DAPI bright heterochromatin clusters (Fig. 6B and C). Such cells could either originate from typical SCs or be present in culture from the start. To determine this, we examined early time points and detected $\mathrm{WT} 1^{+}$cells with atypical nuclei as early as day 1 of culture. From day 1 to day 2, these cells began to incorporate BrdU (Fig. 6D). Their nuclei were similar to
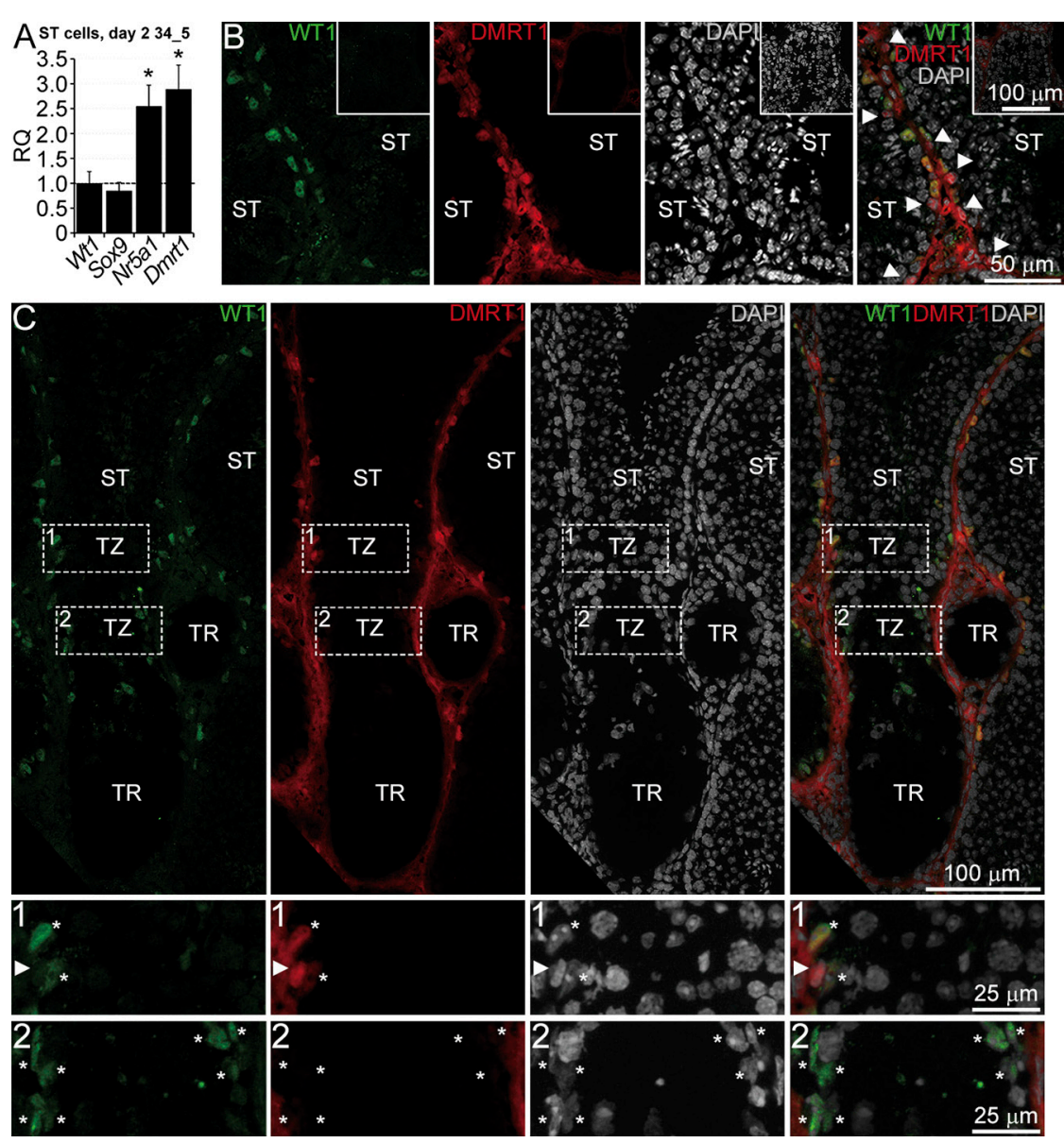

Figure 8 DMRT1 expression in the TZ SCs. (A) qRT-PCR analysis for the indicated genes in the 34_5 TZ and ST cultures on day 2 . Relative quantification of RNA (RQ) was normalized with Wt1. A dotted line indicates levels of gene expression in the TZ SCs. All bars indicate mean \pm S.E.M. ${ }^{*} P<0.05$ (Mann-Whitney $U$ test, $n=3$ ). (B, C) Double immunostaining of seminiferous tubules (ST) (B) and TZ (C) for WT1 and DMRT1. TR tubulus rectus, asterisks indicate SCs, arrowheads point to spermatogonia. $(1,2)$ Enlarged images of areas denoted by the dash boxes in $(\mathrm{C})$. Insets in (B) represent negative controls without first antibodies. 
those of immature SCs (Fig. 6E and F, asterisks). Using live imaging of the TZ culture from day 1 to day 7 , we demonstrated that cells forming $\mathrm{WT}^{+}{ }^{+}$colonies had atypical nuclei from the start of the culture, and SCs exhibiting a typical morphology did not form colonies (Supplementary Fig. 3).

Histological analysis of adult murine testes revealed that in the distal part of the TZ (adjacent to the seminiferous tubules) where some germ cells still occur, SCs are morphologically indistinguishable from SCs of the seminiferous tubules (Fig. 7A, C, and D, asterisks). However, in the proximal part of the TZ, especially in the valve-like terminal segment, some SCs have atypical nuclei with several heterochromatin clusters (Fig. 7A and B, arrowheads). Such modified SCs are positively stained for WT1 (Fig. 7E). Confocal optical serial sections of their nuclei (Fig. 7F) clearly demonstrate their difference from "normal" SCs of the seminiferous tubules (Fig. 7G) and their similarity to the TZ SCs forming colonies in culture (Fig. 6D). However, immunofluorescence analysis of the testes from adult mice treated with BrdU for 5 days did not detect BrdU ${ }^{+} \mathrm{SCs}$ in the TZ (Fig. $7 \mathrm{H}$ ) as well as in the seminiferous tubules.

\section{TZ SCs express DMRT1 at a low level}

To compare ST and TZ SCs, expression of some essential SC transcription factors was measured on day 2 of culture. Dmrt1 as well as Nr5a1 was found to be downregulated in TZ SCs (Fig. 8A). Low DMRT1 expression in the TZ culture was described above (Fig. 3J). Double immunostaining of testis sections for WT1 and DMRT1 confirmed those data. SCs from seminiferous tubules were positively stained for DMRT1 (Fig. 8B) as well as spermatogonia (Fig. 8B, arrowheads). SCs from the part of the TZ where round spermatids were present were DMRT1 positive too (Fig. 8C, 1, asterisks). By contrast, there was no detectable DMRT1 signal in the SCs from the more proximal areas of the TZ (Fig. 8C, 2, asterisks). DMRT1 is essential to maintain testis determination; a complete loss of DMRT1 activates FOXL2, a female-specific transcription factor and reprograms SCs into granulosa cells (Matson et al. 2011). However, we did not observe any positive signal in the TZ SCs when immunostaining testis sections for FOXL2 (Supplementary Fig. 2D and E).

\section{Discussion}

Our results show that most of the SCs isolated from seminiferous tubules remain quiescent in vitro. Only a few of them incorporated BrdU at early time points but did not proliferate more than once or twice; some of them are likely to be cell cycle arrested. Neither higher serum concentration nor changing culture conditions (temperature and oxygen level) enhance their proliferation. This finding is in accordance with previous studies where loss of the tight junction protein claudin 11 (Mazaud-Guittot et al. 2010), the gap junction protein connexin-43 (Sridharan et al. 2007), and retinoblastoma protein (RB), the cell cycle regulator (Nalam et al. 2009), caused mouse ST SCs proliferate in vivo after puberty. However, the number of cycling SCs was extremely low. A few ST SCs enter the cell cycle in human testes after prolonged gonadotropin suppression (Tarulli et al. 2013). In in vitro studies, 17ß-estradiol (Lucas et al. 2008) and activin A (Nicholls et al. 2012) were found to induce active proliferation of SCs from 15- and 20-day-old rats respectively. However, authors isolated cells from the whole testes, so it is not obvious ST or TZ SCs proliferate in culture. Further research is needed to clarify the ability of such factors as activin and estradiol to stimulate ST SC proliferation.

SCs isolated from TZ, by contrast, do actively proliferate in culture and form colonies of epithelial-like cells. Culture conditions affect their growth: the total area of colonies and, correspondingly, the percentage of SCs (WT1+ cells) were higher in the 37_21 culture, whereas in the 34_5 culture, colonies were smaller in size but larger in number. We suggest that under more physiological conditions (34_5 culture), cells attach to the culture surface more efficiently, and therefore more colonies are generated. However, it was previously found that SC growth is highly affected by cell concentration (Schlatt et al. 1996), so at early time points, SCs in the 34_5 culture surrounded by larger amounts of cells proliferate more slowly than SCs in the 37_21 culture. Later, the culture surface is occupied by more numerous PMCs, which also attached more efficiently, and SCs stop proliferating because of contact inhibition.

Immunofluorescence and qRT-PCR analysis of TZ SCs demonstrated that they express Gata4, Wt1, Sox9, Nr5a1, and Dmrt1, which could be considered as key SC transcriptional factors because their concomitant expression is necessary and sufficient for efficient reprogramming of MEFs into Sertoli-like cells (Buganim et al. 2012). The degree of TZ SC differentiation in culture is not obvious; it seems that they have a mixed phenotype. They proliferate like neonatal SCs, display immature nuclear morphology, and generate cord-like structures in the 3D culture. However, at the same time, they highly express the mature SC markers Gata 1 and Trf.

The interesting feature of TZ SCs is the ACTA2 expression that appears in these cells from day 4 of culture. In the postnatal testis, ACTA2 expression is restricted to PMCs and smooth muscle cells of the vascular walls, but in the embryonal development, ACTA2 is expressed at a low level in most interstitial cells of the testis (Cool et al. 2008), which are derived from cells of the coelomic epithelium that earlier gave rise to SCs (Cool et al. 2012). Therefore, ACTA2 expression in TZ SCs could be a sign of their dedifferentiation to the early precursors. 
Table 3 The main characteristics of the two SC populations in the adult mouse testis.

\begin{tabular}{lll}
\hline Characteristics & ST SCs & TZ SCs \\
\hline Proliferation in vitro & Extremely low & High \\
Proliferation in vivo & No & No \\
Nuclear morphology & Typical with two large perinucleolar & Atypical with several smaller heterochromatin \\
& chromocenters & clusters \\
WT1, SOX9, GATA4, and vimentin expression & Yes & Yes \\
DMRT1 expression & High & Low \\
ACTA2 expression in vitro & No & Yes \\
Cord formation & No & Yes \\
\hline
\end{tabular}

Live imaging data of colony formation in the TZ culture and its immunostaining at early time points provided evidence that atypical WT1 ${ }^{+} \mathrm{SC}$ s with several heterochromatin clusters in the nucleus were present from the very start of the culture and gave rise to the colonies. Such SCs were revealed in vivo: in the valvelike terminal segment of TZ just at the border between the seminiferous tubule and the tubulus rectus. They correspond to "modified" SCs described in the early electron microscopic studies (Dym 1974, Osman 1978, Nykänen 1979, Wrobel et al. 1986) and proliferating SCs in Syrian hamster testes (Aiyama et al. 2015). In this study, we did not observe any BrdU ${ }^{+} S C s$ in the TZ in vivo. There may be a principal difference between mouse and hamster TZ SCs: hamster cells proliferate in vivo and then probably become typical SCs that support germ cell development (Aiyama et al. 2015), but mouse cells lost this ability, although they are still able to proliferate in culture. It is also possible that mouse TZ SCs can divide only after testis injury when new SCs are required.

In this study, we paid special attention to one of the key SC transcriptional factors: DMRT1. Its expression in TZ SCs in vivo and at early time points in culture significantly decreased, according to qRT-PCR and immunofluorescence data. As Dmrt1 expression is increased during SC maturation (Chen \& Heckert 2001), and SCs from mice with knockout of Dmrt1 did not differentiate properly during postnatal development and fail to cease proliferation (Raymond et al. 2000, Fahrioglu et al. 2007), we speculate that the ability TZ SCs to proliferate in culture and exhibit other immature characteristics may be caused by decreased DMRT1 levels.

In conclusion, our results indicate the presence of two SC populations, ST and TZ SCs, in the adult testis, whose main characteristics are summarized in Table 3. It is obvious that TZ SCs are not completely differentiated. The in vivo role of this population is not clear, but it may contribute to the restoration of SC number after testicular injury and during aging. Such SC renewal could even occur under normal circumstances, as indirect data from Syrian hamster testes suggest (Aiyama et al. 2015). Besides its fundamental significance, our finding could be important for medical science. It is known that alterations in SC function and SC loss lead to impaired spermatogenesis and irreversible infertility (Salameh \& Swerdloff 2005). For example, we generated a mouse model of viral testicular infection that mimics human orchitis and demonstrated massive SC death and subsequent infertility (Malolina et al. 2014). Such spermatogenic defects could be corrected with combined transplantation of SCs and germ cells (Shinohara et al. 2003, Zhang et al. 2009, Malolina et al. 2016). Furthermore, SCs were demonstrated to support and protect nontesticular cell grafts, such as pancreatic islet cells and neurons (Mital et al. 2010). A limiting factor for using SCs for cell therapy was that immature SCs suitable for transplantation can only be obtained from neonatal donors. However, in this study, we demonstrated that adult SCs from TZ can be an alternative source of immature SCs and hence may be applied to clinical applications in the future.

\section{Supplementary data}

This is linked to the online version of the paper at http://dx.doi. org/10.1530/REP-16-0013.

\section{Declaration of interest}

The authors declare that there is no conflict of interest that could be perceived as prejudicing the impartiality of the research reported.

\section{Funding}

The study was supported by the grant of the Presidium RAS Program "Fundamental research for biomedical technologies" and the grant from the Russian Foundation for Basic Research (RFBR), research project no. 16-34-60119. The research was done using equipment of the Core Centrum of Insitute of Developmental Biology RAS.

\section{References}

Ahmed EA, Barten-van Rijbroek AD, Kal HB, Sadri-Ardekani H, Mizrak SC, van Pelt AM \& de Rooij DG 2009 Proliferative activity in vitro and DNA repair indicate that adult mouse and human Sertoli cells are not terminally differentiated, quiescent cells. Biology of Reproduction $\mathbf{8 0}$ 1084-1091. (doi:10.1095/biolreprod.108.071662)

Aiyama Y, Tsunekawa N, Kishi K, Kawasumi M, Suzuki $H$, Kanai-Azuma M, Kurohmaru M \& Kanai Y 2015 A Niche for GFR $\alpha 1$ positive spermatogonia in the terminal segments of the seminiferous tubules in Hamster testes. Stem Cells 33 2811-2824. (doi:10.1002/stem.v33.9) 
Buganim Y, Itskovich E, Hu YC, Cheng AW, Ganz K, Sarkar S, Fu D, Welstead GG, Page DC \& Jaenisch R 2012 Direct reprogramming of fibroblasts into embryonic Sertoli-like cells by defined factors. Cell Stem Cell 11 373-386. (doi:10.1016/j.stem.2012.07.019)

Carpenter AE, Jones TR, Lamprecht MR, Clarke C, Kang IH, Friman O, Guertin DA, Chang JH, Lindquist RA, Moffat J et al. 2006 CellProfiler: image analysis software for identifying and quantifying cell phenotypes. Genome Biology 7 R100. (doi:10.1186/gb-2006-7-10-r100)

Chen JK \& Heckert LL 2001 Dmrt1 expression is regulated by folliclestimulating hormone and phorbol esters in postnatal Sertoli cells. Endocrinology 142 1167-1178. (doi: 10.1210/en.142.3.1167)

Cool J, Carmona FD, Szucsik JC \& Capel B 2008 Peritubular myoid cells are not the migrating population required for testis cord formation in the XY gonad. Sexual Development 2 128-133. (doi:10.1159/000143430)

Cool J, DeFalco T \& Capel B 2012 Testis formation in the fetal mouse: dynamic and complex de novo tubulogenesis. Wiley Interdisciplinary Reviews: Developmental Biology 1 847-859. (doi:10.1002/wdev.v1.6)

Cupp AS \& Skinner MK 2005 Embryonic Sertoli cell differentiation. In Sertoli Cell Biology, edn 1, pp 43-70. Eds MK Skinner \& MD Griswold. San Diego and London: Elsevier Academic Press.

Dym M 1974 The fine structure of monkey Sertoli cells in the transitional zone at the junction of the seminiferous tubules with the tubuli recti American Journal of Anatomy 140 1-25. (doi:10.1002/(ISSN)1553-0795)

Fahrioglu U, Murphy MW, Zarkower D \& Bardwell VJ 2007 mRNA expression analysis and the molecular basis of neonatal testis defects in Dmrt1 mutant mice. Sexual Development 1 42-58. (doi:10.1159/000096238)

Harrison RG \& Weiner JS 1949 Vascular patterns of the mammalian testis and their functional significance. Journal of Experimental Biology 26 304-316.

Livak KJ \& Schmittgen TD 2001 Analysis of relative gene expression data using real-time quantitative PCR and the 2(-Delta Delta $\mathrm{C}(\mathrm{T})$ ) method. Methods 4 402-408. (doi:10.1006/meth.2001.1262)

Lucas TF, Siu ER, Esteves CA, Monteiro HP, Oliveira CA, Porto CS \& Lazari MF 2008 17beta-estradiol induces the translocation of the estrogen receptors ESR1 and ESR2 to the cell membrane, MAPK3/1 phosphorylation and proliferation of cultured immature rat Sertoli cells. Biology of Reproduction 78 101-114. (doi:10.1095/ biolreprod.107.063909)

Malolina EA, Kulibin AYu, Naumenko VA, Gushchina EA, Zavalishina LE \& Kushch AA 2014 Herpes simplex virus inoculation in murine rete testis results in irreversible testicular damage. International Journal of Experimental Pathology 95 120-130. (doi:10.1111/iep.2014.95. issue-2)

Malolina EA, Kulibin AY \& Kushch AA 2016 Neonatal testicular cell transplantation restores murine spermatogenesis damaged in the course of herpes simplex virus-induced orchitis. Reproduction, Fertility and Development 28 757-764. (doi:10.1071/RD14255)

Matson CK, Murphy MW, Sarver AL, Griswold MD, Bardwell VJ \& Zarkower D 2011 DMRT1 prevents female reprogramming in the postnatal mammalian testis. Nature 476 101-104. (doi:10.1038/ nature10239)

Mazaud-Guittot S, Meugnier E, Pesenti S, Wu X, Vidal H, Gow A \& Le Magueresse-Battistoni B 2010 Claudin 11 deficiency in mice results in loss of the Sertoli cell epithelial phenotype in the testis. Biology of Reproduction 82 202-213. (doi:10.1095/biolreprod.109.078907)

Mital P, Kaur G \& Dufour JM 2010 Immunoprotective sertoli cells: making allogeneic and xenogeneic transplantation feasible. Reproduction 139 495-504. (doi:10.1530/REP-09-0384)

Naito M, Terayama H, Hirai S, Qu N, Kawata S \& Itoh M 2009 Histopathology of the tubuli recti at the start of experimental autoimmune orchitis in mice. Medical Molecular Morphology 42 230-235. (doi:10.1007/ s00795-009-0469-4)

Nalam RL, Andreu-Vieyra C, Braun RE, Akiyama H \& Matzuk MM 2009 Retinoblastoma protein plays multiple essential roles in the terminal differentiation of Sertoli cells. Molecular Endocrinology 23 1900-1913. (doi:10.1210/me.2009-0184)
Nicholls PK, Stanton PG, Chen JL, Olcorn JS, Haverfield JT, Qian H, Walton KL, Gregorevic P \& Harrison CA 2012 Activin signaling regulates Sertoli cell differentiation and function. Endocrinology 153 6065-6077. (doi:10.1210/en.2012-1821)

Nykänen M 1979 Fine structure of the transitional zone of the rat seminiferous tubule. Cell and Tissue Research 198 441-454. (doi:10.1007/BF00234189)

Osman DI 1978 On the ultrastructure of modified Sertoli cells in the terminal segment of seminiferous tubules in the boar. Journal of Anatomy 127 603-613.

Raymond CS, Murphy MW, O'Sullivan MG, Bardwell VJ \& Zarkower D 2000 Dmrt1, a gene related to worm and fly sexual regulators, is required for mammalian testis differentiation. Genes and Development 14 2587-2595. (doi:10.1101/gad.834100)

Salameh WA \& Swerdloff RS 2005 Conditions affecting Sertoli cells. In Sertoli Cell Biology, edn 1, pp 383-413. Eds MK Skinner \& MD Griswold. San Diego and London: Elsevier Academic Press.

Sang QX, Dym M \& Byers SW 1990 Secreted metalloproteinases in testicular cell culture. Biology of Reproduction 43 946-955. (doi:10.1095/biolreprod43.6.946)

Schlatt S, de Kretser DM \& Loveland KL 1996 Discriminative analysis of rat Sertoli and peritubular cells and their proliferation in vitro: evidence for follicle-stimulating hormone-mediated contact inhibition of Sertoli cell mitosis. Biology of Reproduction 55 227-235. (doi:10.1095/ biolreprod55.2.227)

Shinohara T, Orwig KE, Avarbock MR \& Brinster RL 2003 Restoration of spermatogenesis in infertile mice by Sertoli cell transplantation. Biology of Reproduction 68 1064-1071. (doi:10.1095/biolreprod.102.009977)

Sridharan S, Simon L, Meling DD, Cyr DG, Gutstein DE, Fishman GI, Guillou F \& Cooke PS 2007 Proliferation of adult sertoli cells following conditional knockout of the Gap junctional protein GJA1 (connexin 43) in mice. Biology of Reproduction 76 804-812. (doi:10.1095/ biolreprod.106.059212)

Tarulli GA, Stanton PG, Lerchl A \& Meachem SJ 2006 Adult sertoli cells are not terminally differentiated in the Djungarian hamster: effect of FSH on proliferation and junction protein organization. Biology of Reproduction 74 798-806. (doi:10.1095/biolreprod.105.050450)

Tarulli GA, Stanton PG \& Meachem SJ 2012 Is the adult Sertoli cell terminally differentiated? Biology of Reproduction 87 1-11. (doi:10.1095/ biolreprod.112.101691)

Tarulli GA, Stanton PG, Loveland KL, Rajpert-De Meyts E, McLachlan RI \& Meachem SJ 2013 A survey of Sertoli cell differentiation in men after gonadotropin suppression and in testicular cancer. Spermatogenesis 3 e24014. (doi: 10.4161/spmg.24014)

Vaupel P, Kallinowski F \& Okunieff P 1989 Blood flow, oxygen and nutrient supply, and metabolic microenvironment of human tumors: a review. Cancer Research 49 6449-6465.

Wrobel KH, Schilling E \& Zwack M 1986 Postnatal development of the connexion between tubulus seminiferous and tubulus rectus in the bovine testis. Cell and Tissue Research 246 387-400. (doi:10.1007/ BF00215902)

Zhang Z, Shao S, Shetty G \& Meistrich ML 2009 Donor Sertoli cells transplanted into irradiated rat testes stimulate partial recovery of endogenous spermatogenesis. Reproduction 137 497-508. (doi:10.1530/REP-08-0120)

Zhang J, Hatakeyama J, Eto K \& Abe S 2014 Reconstruction of a seminiferous tubule-like structure in a 3 dimensional culture system of re-aggregated mouse neonatal testicular cells within a collagen matrix. General and Comparative Endocrinology 205 121-132. (doi:10.1016/j. ygcen.2014.03.030)

Received 10 January 2016

First decision 12 February 2016

Revised manuscript received 5 June 2016

Accepted 4 July 2016 